\title{
Interrelationship between tetracycline resistance determinants, phylogenetic group affiliation and carriage of class 1 integrons in commensal Escherichia coli isolates from cattle farms
}

\author{
Kuastros Mekonnen Belaynehe, Seung Won Shin and Han Sang Yoo *i]
}

\begin{abstract}
Background: Carriage of antibiotic-resistant foodborne pathogens by food production animals is one of many contributors to treatment failure in health care settings, and it necessitates an integrated approach to investigate the carriage of resistant pathogens harboring integrons in food-producing animals.

Methods: Escherichia coli isolates with reduced susceptibility to tetracycline antibiotics $(n=92)$ were tested for associations between carriage of class 1 integrons, phylogenetic group affiliation and tetracycline resistance determinants using the MIC method, PFGE analysis, PCR and sequencing.

Results: Phylogroups B1 and A were the most common (58.7 and 19.6\%, respectively), followed by groups D (20.7\%) and B2 (1.1\%). All isolates carried at least one of the tet genes examined. In addition, 88 (95.7\%) of all tetracycline-resistant isolates carried tet(A) or tet(B), while $47(51.1 \%)$ and $41(44.6 \%)$ harbored only tet(A) or tet(B), respectively. Likewise, isolates harboring these genes had a higher chance $(P<0.05)$ of carrying class 1 integrons. Of the tested isolates, 38 (41.3\%) carried the int/1 gene. Classical integrons with complete genes (sull and qacE $\triangle 1$ ) at the $3^{\prime}$-CS were recognized in 27 isolates. PCR screening and subsequent sequencing demonstrated that $84.2 \%$ (32/38) of the int/1-positive isolates harbored resistance gene cassettes. Overall, seven gene cassettes were identified, either solely or combined with another gene cassette. The most common gene was aadA1 (10 isolates), followed by a combination of aadA1-dfrA1 (seven isolates), aadA1dfrA12 (six isolates) and aadA1-aadA2-dfrA12 (three isolates). Genetic typing using PFGE showed minimum clonal relatedness with 28 different clusters and 12-25 discernible DNA fragments.
\end{abstract}

Conclusions: This study brings new insight into the relationships between the presence of integrons, phylogenetic group association and characteristics of tetracycline antibiotic resistance determinants in commensal E. coli strains.

Keywords: E. coli, Class 1 integrons, Tetracycline resistance, Phylogenetic group, PFGE

* Correspondence: yoohs@snu.ac.kr

Department of Infectious Diseases, College of Veterinary Medicine, Seoul

National University, Seoul 08826, Republic of Korea

(C) The Author(s). 2018 Open Access This article is distributed under the terms of the Creative Commons Attribution 4.0 International License (http://creativecommons.org/licenses/by/4.0/), which permits unrestricted use, distribution, and reproduction in any medium, provided you give appropriate credit to the original author(s) and the source, provide a link to the Creative Commons license, and indicate if changes were made. The Creative Commons Public Domain Dedication waiver (http://creativecommons.org/publicdomain/zero/1.0/) applies to the data made available in this article, unless otherwise stated. 


\section{Background}

The spread and emergence of resistance to antimicrobial drugs among bacteria has been observed over the past several decades, and this constraint has been a constant impediment to effective infectious disease therapy for as long as antibiotics have been used [1]. In many cases, multidrug resistance was determined to be associated with transmissible plasmids, and the importance of integrons in the acquisition of resistance genes constitute the major vector of multidrug resistance in Gram-negative and, to a lesser extent, in Gram-positive bacteria [2-4].

Over the last few years, rigorous exploration of the diversity of integrons in natural environments has indicated that they are more than just a curious feature of antibiotic-resistant pathogens but that they play a more general and crucial role in the genomic evolution and adaptation of bacteria [5]. To date, five mobile integron classes have been described and characterized based on variations in the intI sequences. However, class 1 integrons are ubiquitous and the most frequently encountered among clinical and commensal isolates; therefore, they have been the focus of numerous studies $[1,2,6]$.

The basic structure of class 1 integrons includes two conserved segments (CSs) that are usually separated by a variable region that includes mobile cassettes containing antibiotic resistance genes. The $5^{\prime}$-CS carries an integrase class 1 (intI1) gene encoding an integrase enzyme and a recombination site (attI1), whereas qacEA1 and sul1, which confer resistance to quaternary ammonium compounds and sulfonamides, respectively, are localized at the $3^{\prime}-\mathrm{CS}[1,6-8]$. The site-specific recombination system between attI and $a t t C$ has enabled a diverse array of resistance determinants to be drawn by individual class 1 integrons [1, 2, 9].

The coding regions of the gene cassettes have no promoters; however, most cassettes encode various antimicrobial resistance genes, with more than 130 distinguishable resistance genes having been found to date [6]. The majority of class 1 integrons harbors an aminoglycoside adenyltransferase gene ( $a a d A)$ and a dihydrofolate reductase gene $(d f r)$, which confer resistance to streptomycin and spectinomycin, and trimethoprim, respectively $[10,11]$.

Tetracycline has been used in human and veterinary medicine and as a growth promoter in animal husbandry. The major mechanisms of tetracycline resistance in Enterobacteriaceae are mediated through one of several mechanisms; namely, efflux pump activity, ribosomal protection, and enzymatic inactivation. The predominant genes that confer tetracycline resistance via efflux pump activity are $\operatorname{tet}(A), \operatorname{tet}(B), \operatorname{tet}(C), \operatorname{tet}(D)$, tet $(E)$, and tet $(G)$. Indiscriminate application of tetracyclines in food-producing animals enhances multidrug resistance due to antibiotic selective pressure induced by the presence of high environmental concentrations of the antibiotics. This selective pressure ultimately leads to increased prevalence of tetracycline resistance via the tet genes and promotes the dissemination of mobile genetic elements in bacteria $[12,13]$.

E. coli isolates belong to four major phylogenetic groups, A, B1, B2, and D, and strains from the B2 phylogenetic group happen to be the least resistant to antimicrobials. Moreover, there is a tendency towards lower integron carriage among phylogroup B2 [14]. Nevertheless, due to certain factors, such as the level of resistance to antimicrobials, the site of the infection and geographical location, there is variation in the prevalence of different phylogenetic groups [15]. Isolates in the phylogenetic groups $\mathrm{B} 1$ and $\mathrm{D}$ tend to harbor class 1 integrons, and a previous report also showed that intI-positive B2 strains were the least prevalent [16]. There are various observations on the interrelationship of different phylogroups and integron carriage for environmental, human and animal isolates raising the hypothesis that the two phenomena are connected and indicating that various genetic elements are involved in strains with different phenotypes. Characterization of this association will help to better understand the infection process and will reflect the possible different survival strategies of $E$. coli phylogroups under different circumstances.

Antimicrobial resistant bacteria derived from animals seriously compromises public health by causing food-borne infections and raises a food safety issue globally. The effects of such bacteria are not only limited to food safety but also pose occupational hazards for animal handlers, meat inspectors and veterinarians. In particular, carriage of antibiotic-resistant foodborne pathogens by food-production animals is one of many contributors to treatment failure in health care settings, and it establishes the need for a detailed and thorough investigation of the carriage of such antibiotic-resistant pathogens harboring integrons in food-producing animals [12]. Furthermore, integrons are not only limited to pathogenic organisms but have also been isolated from bacteria recovered from environmental samples and healthy animals [17]. Similarly, the lack of sufficient and current information describing the association between antibiotic resistance and phylogenic groups with respect to integron carriage in commensal $E$. coli isolates of cattle from Korea necessitates further research. Therefore, in the present study, we investigated the role of integrons and their associated diverse gene cassettes in mediating antimicrobial resistance in commensal $E$. coli isolates recovered from cattle. Moreover, we examined the relationship between class 1 integron 
carriage with respect to phylogroups and patterns of tetracycline resistance.

\section{Methods}

\section{Selection of bacterial strains for the study}

In total, 247 commensal $E$. coli isolates obtained by our research group between 2014 and 2015 from fecal samples from 405 tested animals at four healthy beef cattle farms located in four different cities in South Korea (Pyeongchang, Anyang, Yangpyeong, and Cheonan) were used in the present study [18]. The beef farms consisted of different age groups of cattle, such as weaned calves, bulls and steers. Since the farms are intensive, cattle were kept in confinement in a conventional housing system. Fecal samples were freshly collected from the rectum of each cattle and a single bacterial isolate was recovered per animal. All bacterial strains were routinely cultured in tryptic soy broth (TSB) (Oxoid, Basingstoke, UK) for $18 \mathrm{~h}$ at $37{ }^{\circ} \mathrm{C}$. Among the 247 isolates, $92 \mathrm{E}$. coli isolates demonstrating resistance or decreased susceptibility by microbroth dilution assays to any of the tetracycline antibiotics referred to below were selected for further investigation.

\section{Susceptibility testing}

Phenotypic characterization for all isolates was performed using the disc diffusion method, and the following antibiotic discs were analyzed in this study: tetracycline (TE, $30 \mu \mathrm{g})$, streptomycin $(\mathrm{S}, 10 \mu \mathrm{g})$, chloramphenicol (C, $30 \mu \mathrm{g})$, ampicillin (AMP, $10 \mu \mathrm{g}$ ), amoxicillin-clavulanic acid (AMC, $30 \mu \mathrm{g}$ ), ciprofloxacin (CIP, $5 \mu \mathrm{g})$, nalidixic acid (NA, $30 \mu \mathrm{g})$ and trimethoprim-sulfamethoxazole (SXT, $25 \mu \mathrm{g}$ ) (Sigma-Aldrich, St. Louis, MO, USA). The MICs for oxytetracycline, doxycycline, tetracycline, minocycline and tigecycline were determined using cation-adjusted Mueller-Hinton broth (Oxoid, Basingstoke, UK). All susceptibility testing was performed according to the procedures and interpretive criteria specified by the Clinical Laboratory Standards Institute (CLSI), and E. coli ATCC 25922 was used as a quality control strain [19].

\section{Phylogenetic group determination}

E. coli phylogenetic groups (A, B1, B2 and D) were investigated by amplifying two genes and a DNA fragment using multiplex PCR as previously described [20].

\section{Analysis of antimicrobial resistance genes}

PCR amplification to investigate the tetracycline resistance-encoding genes was conducted for all isolates. The following genes, encoding the tetracycline efflux mechanism, were investigated as previously described: $\operatorname{tet}(A), \operatorname{tet}(B), \operatorname{tet}(C), \operatorname{tet}(D), \operatorname{tet}(E)$ and $\operatorname{tet}(G)[12,21-24]$. Furthermore, genes conferring resistance to sulfonamide antibiotics (sul1, sul2 and sul3) and genes conferring chloramphenicol/florfenicol resistance (cat1, cmlA and floR) were also analyzed. PCR amplification of the resistance genes was conducted using the primers presented in Table 1 [12, 21-30].

\section{Detection and characterization of class 1 integrons and their gene cassettes}

Total DNA was extracted by boiling a suspension of overnight-cultured bacterial cells [grown on tryptic soy agar plates (TSA) at $37^{\circ} \mathrm{C}$ for $10 \mathrm{~min}$ ] in $200 \mu \mathrm{l}$ of sterile RNase/DNase-free distilled water. All E. coli isolates were PCR screened for the presence of IntI1 gene-encoding class 1 integrons. Further testing was performed on the integron positive isolates for the presence of gene cassettes in the variable region and the sul1 and qacE $\Delta 1$ genes in the $3^{\prime}$-CS. All primers and PCR conditions are presented in Table 1 . Gel purification of all PCR products was conducted using PCR quick-spin PCR product purification kits (iNtRON Biotechnology, USA), after which the samples were sequenced (Macrogen Co., Seoul, Korea). Following sequencing, the gene cassettes within the variable regions of the class 1 integrons were determined by using BLAST (Basic Local Alignment Search Tool) searches of the NCBI database (National Center for Biotechnology Information).

\section{Clonal relationships among integron positive strains}

Determination of the genetic relationship between the integron positive isolates was accomplished by pulsed-field gel electrophoresis (PFGE) analysis according to the protocols and criteria previously established by the Centers for Disease Control and Prevention (CDC) using $\mathrm{XbaI}$ as the restriction enzyme. Briefly, following 18 to $20 \mathrm{~h}$ growth on TSA at $37{ }^{\circ} \mathrm{C}$, genomic DNA was digested with $50 \mathrm{U}$ XbaI (TaKaRa, Japan) for $2 \mathrm{~h}$ at $37{ }^{\circ} \mathrm{C}$, then the DNA fragments were subsequently separated on a 1.0\% SeaKem Gold agarose gel (Lonza, USA) in 0.5× Tris-borate-EDTA (TBE) buffer using a CHEFMapper gel apparatus (Bio-Rad Laboratories, California, USA). The conditions for electrophoresis were as follows: pulse time, $2-30 \mathrm{~s}$ at $14{ }^{\circ} \mathrm{C}$; run time, $18 \mathrm{~h}$; voltage, $6 \mathrm{~V} / \mathrm{cm}$. Analysis of the image was performed by using the Bionumerics software (Applied Maths, Belgium).

\section{Statistical analysis}

All experiment data are stored in Excel 2010, and the susceptibility testing was analyzed using IBM SPSS/Statistics, version 24. The association between the tet genes and the presence of class 1 integron gene was analyzed by Fisher's exact test or Pearson's $x^{2}$ test, contingent on cell frequencies. The median MICs for the respective 
Table 1 Primers used for the PCR detection of resistance genes

\begin{tabular}{|c|c|c|c|c|c|}
\hline Primer name & Target gene & Nucleotide sequence & Annealing temperature $\left({ }^{\circ} \mathrm{C}\right)$ & Amplicon size (bp) & Reference \\
\hline TetA-F & $\operatorname{tet}(A)$ & GGCGGTCTTCTTCATCATGC & 55 & 502 & [21] \\
\hline TetA-R & & CGGCAGGCAGAGCAAGTAGA & & & \\
\hline TetB-F & $\operatorname{tet}(B)$ & CATTAATAGGCGCATCGCTG & 55 & 930 & [21] \\
\hline TetB-R & & TGAAGGTCATCGATAGCAGG & & & \\
\hline TetC-F & $\operatorname{tet}(C)$ & GCTGTAGGCATAGGCTTGGT & 55 & 888 & [21] \\
\hline TetC-R & & GCCGGAAGCGAGAAGAATCA & & & \\
\hline TetD-F & $\operatorname{tet}(D)$ & GAGCGTACCGCCTGGTTC & 55 & 780 & [12] \\
\hline TetD-R & & TCTGATCAGCAGACAGATTGC & & & \\
\hline TetE-F & $\operatorname{tet}(E)$ & AAACCACATCCTCCATACGC & 55 & 278 & [22] \\
\hline TetE-R & & AAATAGGCCACAACCGTCAG & & & \\
\hline TetG-F & $\operatorname{tet}(G)$ & GCTCGGTGGTATCTCTGCTC & 55 & 468 & [23] \\
\hline TetG-R & & AGCAACAGAATCGGGAACAC & & & \\
\hline Sul1-F & sul1 & CGGCGTGGGCTACCTGAACG & 57 & 433 & [24] \\
\hline Sul1-R & & GCCGATCGCGTGAAGTTCCG & & & \\
\hline Sul2-F & sul2 & CGGCATCGTCAACATAACCT & 57 & 721 & [21] \\
\hline Sul2-R & & TGTGCGGATGAAGTCAGCTC & & & \\
\hline Sul3-F & sul3 & CAACGGAAGTGGGCGTTGTGGA & 57 & 244 & [25] \\
\hline Sul3-R & & GCTGCACCAATTCGCTGAACG & & & \\
\hline Cat-F & Cat & GGT GAG CTG GTG ATA TGG & 55 & 209 & [26] \\
\hline Cat-R & & GGG ATT GGC TGA GAC GA & & & \\
\hline Flor $-\mathrm{F}$ & flor & CAC GTT GAG CCT CTA TAT & 55 & 868 & [27] \\
\hline Flor $-\mathrm{R}$ & & ATG CAG AAG TAG AAC GCG & & & \\
\hline $\mathrm{CmlA}-\mathrm{F}$ & $\mathrm{cm} / \mathrm{A}$ & TGT CAT TTA CGG CAT ACT CG & 55 & 455 & [27] \\
\hline $\mathrm{CmlA}-\mathrm{F}$ & & ATC AGG CAT CCC ATT CCC AT & & & \\
\hline Var1-F & varl & GGCATCCAAGCAGCAAG & 55 & Variable & [28] \\
\hline Var1-R & & AAGCAGACTTGACCTGA & & & \\
\hline qacE $\Delta 1 \mathrm{~F}$ & qacE $\Delta 1$ & ATCGCAATAGTTGGCGAAGT & 60 & 225 & [29] \\
\hline qacE $\Delta 1 \mathrm{R}$ & & CAAGCTITTGCCCATGAAGC & & & \\
\hline Intl1-F & int/1 & GGGTCAAGGATCTGGATTTCG & 60 & 483 & [30] \\
\hline Int11-R & & ACATGCGTGTAAATCATCGTCG & & & \\
\hline
\end{tabular}

tetracycline antibiotics between the isolates with and without Intl1 was analyzed using the Mann-Whitney test. A $P<0.05$ was considered to indicate statistical significance.

\section{Results}

\section{Antimicrobial resistance phenotypes}

The resistance percentages to the tested antibiotics were as follows: streptomycin, 84 (91.3\%); nalidixic acid, 36 (39.1\%); ampicillin, 35 (38\%); chloramphenicol, 28 (30.4\%); trimethoprim-sulfamethoxazole, 24 (26.1\%); ciprofloxacin, 11 (12\%) and amoxicillin-clavulanic, 2 (2.2\%) (Fig. 1). The MIC range for the 92 tetracycline resistant isolates was $>256 \mu \mathrm{g} / \mathrm{ml}$ to $16 \mu \mathrm{g} / \mathrm{ml}$, and their $\mathrm{MIC}_{50}$ and $\mathrm{MIC}_{90}$ values were 128 and $256 \mu \mathrm{g} / \mathrm{ml}$, respectively. Oxytetracycline resistance was identified in all isolates
(MIC range $>256 \mu \mathrm{g} / \mathrm{ml}-32 \mu \mathrm{g} / \mathrm{ml}$ ), of which 49 isolates were highly resistant ( $\mathrm{MIC} \geq 256 \mu \mathrm{g} / \mathrm{ml}$ ). Moreover, 80 strains $(87 \%)$ were resistant to doxycycline, $41(44.6 \%)$ to minocycline and none to tigecycline. Significantly higher median oxytetracycline MICs were observed for isolates with class 1 integrons than for isolates without class 1 integrons $(P<0.006$; Table 2$)$; however, there were no significant statistical differences in the median MIC values for tetracycline, doxycycline, minocycline and tigecycline between class 1 integron-positive and integron-negative strains.

\section{E. coli phylogenetic groups}

Of the 92 isolates, phylogenetic groups $\mathrm{B} 1$ and $\mathrm{D}$ were the most common (54 isolates; $58.7 \%$ and 19 isolates; $20.7 \%$, respectively), followed by group A, which was 


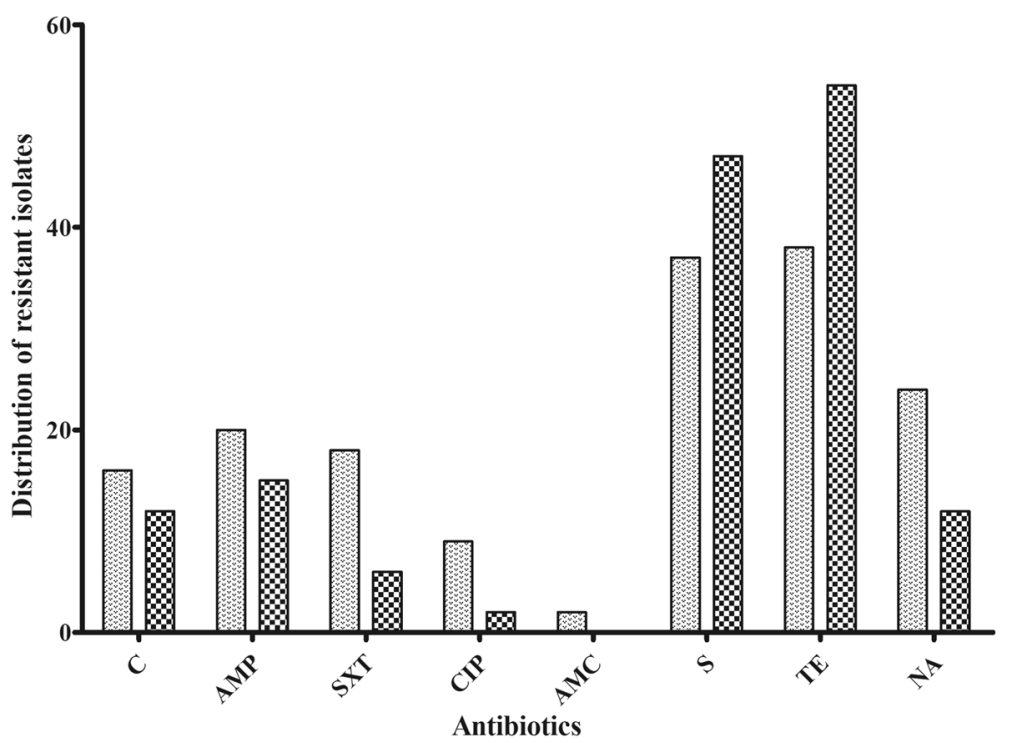

Fig. 1 Different classes of antibiotics with respect to their presence or absence in integrons

assigned to 18 isolates (19.6\%). Group B2 was rare, occurring in only 1 isolate. We also compared integron-positive and integron-negative isolates across the phylogenetic groups, and phylogenetic group D (16 isolates) was the most prevalent among the intI1-positive isolates, whereas B1 (40 isolates) was most prevalent among the intI1-negative isolates. Our results showed an association between the presences of class 1 integrons and affiliation with phylogenetic groups D and B1 $(P<$ 0.01). The frequencies of integron-negative and integron-positive strains for in A and B2 phylogenetic groups were similar, with no statistically significant differences (Fig. 2).

\section{Characterization of antimicrobial resistance genes}

All isolates carried at least one of the tet genes examined. Overall, 88 (95.7\%) of the tetracycline-resistant isolates carried tet(A) or tet(B), with $47(51.1 \%)$ and 41
(44.6\%) isolates harboring only tet(A) or tet(B), respectively. The $\operatorname{tet}(C)$ and $\operatorname{tet}(G)$ genes were found in only five $(5.4 \%)$ and six $(6.5 \%)$ isolates, respectively, and the tet $(C)$ gene was found in isolates that were not harboring integrons. Moreover, eight isolates harbored two tet genes, while none of the isolates carried the tet $(D)$ or tet $(E)$ genes. The distribution of tetracycline resistance genes among the integron-positive and -negative isolates is shown in Table 3. E. coli isolates carrying class 1 integrons were more likely to harbor the $\operatorname{tet}(A)$ gene $(P$ $<0.01)$. In addition, the following determinants for chloramphenicol/florfenicol resistance were identified: cat1 (47.4\%), floR (50\%), and cmlA (18.4\%). Among the $92 \mathrm{E}$. coli isolates investigated, 28 isolates harboring integrons and eight isolates without integrons $(n=36$; $39.1 \%)$ had the sul1 gene. Moreover, the sul2 and sul3 genes were identified in $16(17.4 \%)$ and seven (7.6\%) isolates, respectively.

Table 2 Susceptibility to various tetracycline antibiotics stratified by the presence or absence of class 1 integrons

\begin{tabular}{|c|c|c|c|c|c|c|c|c|c|c|c|}
\hline \multirow{3}{*}{$\begin{array}{l}\text { Antimicrobial } \\
\text { agents }\end{array}$} & \multicolumn{5}{|c|}{ Int/1 present } & \multicolumn{5}{|c|}{ Int/1 Absent } & \multirow[t]{3}{*}{$P$ value } \\
\hline & \multicolumn{4}{|c|}{$\overline{M I C}(\mu \mathrm{g} / \mathrm{ml})$} & \multirow{2}{*}{$\begin{array}{l}\text { Number } \\
(\%) \text { of } \\
\text { resistant } \\
\text { isolates }\end{array}$} & \multicolumn{4}{|c|}{$\overline{M I C}(\mu \mathrm{g} / \mathrm{ml})$} & \multirow{2}{*}{$\begin{array}{l}\text { Number } \\
(\%) \text { of } \\
\text { resistant } \\
\text { isolates }\end{array}$} & \\
\hline & Range & & $\mathrm{MIC}_{50}$ & $\mathrm{MIC}_{90}$ & & Range & & $\mathrm{MIC}_{50}$ & $\mathrm{MIC}_{90}$ & & \\
\hline Tetracycline & 64 & $>256$ & 128 & 256 & $38(41.3)$ & 16 & $>256$ & 128 & 256 & $54(58.7)$ & 0.244 \\
\hline Doxycycline & 4 & 128 & 16 & 64 & $36(39.1)$ & 4 & 64 & 32 & 64 & $44(47.8)$ & 0.975 \\
\hline Oxytetracycline & 64 & $>256$ & $>256$ & $>256$ & $38(41.3)$ & 32 & $>256$ & 256 & $>256$ & $54(58.7)$ & $P<0.006$ \\
\hline Minocycline & 4 & 64 & 8 & 32 & $14(15.2)$ & 2 & 64 & 16 & 32 & $28(30.4)$ & 0.267 \\
\hline Tigecycline & 0.25 & 2 & 1 & 1 & - & 0.25 & 8 & 0.5 & 1 & $1(1.1)$ & 0.054 \\
\hline
\end{tabular}

Numbers indicated in italics indicate significance difference $(P<0.05)$ 


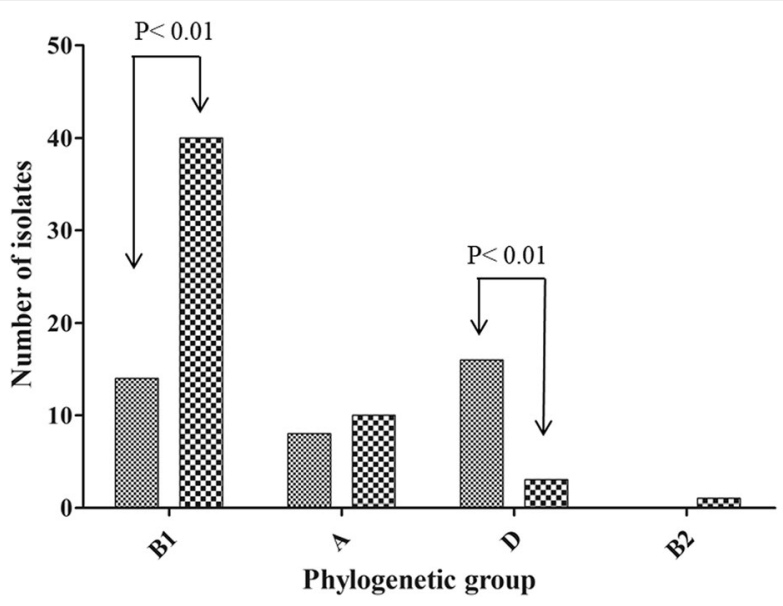

IntIl Positive

8 IntIl Negative

Detection of the int/1 gene and characterization of gene cassettes in the $E$. coli strains

Integrase gene-encoding class 1 integrons were detected by PCR in 38 (41.3\%) isolates. Resistance to quaternary ammonium compounds and sulfonamides conferred by the qacE $\Delta 1$ and sul1 genes in the 3'-CS, respectively, was identified in 36 integron-positive isolates. Among these, 27 contained the entire 3'-CS (qacEA1-sul1) structure, whereas nonclassical integrons lacking the 3 '-CS were found in only two of the 38 intI1-positive $E$. coli isolates. Of the 38 isolates, one had only sul1 in the 3 '-CS and eight possessed only qacEA1 in the 3 '-CS. The class 1 integron variable regions were amplified in $32(84.2 \%)$ of the 38 intI1-positive isolates, and their genetic contents were ascertained via PCR amplification of the integron variable regions and subsequent full sequence analysis. Different lengths of PCR products ranging from $\sim 1-2.5 \mathrm{~kb}$ were observed for strains having variable regions. Of these, the predominant cassette amplicons carried by the isolates were $1 \mathrm{~kb}$ in 18 strains, $1.5 \mathrm{~kb}$ in 10 strains and $2.5 \mathrm{~kb}$ in four strains (Table 4).

Overall, seven gene cassettes and eight distinct profiles of gene cassette arrays, namely, aadA1 (10 isolates),

Table 3 Association between integron-positive and integronnegative $E$. coli isolates and the frequencies of tet genes

\begin{tabular}{llll}
\hline tet genes & \multicolumn{2}{l}{ Class 1 integron presence } & $P$ value \\
\cline { 2 - 3 } & int11 positive & int1 negative & \\
\hline tet $(A)$ & 26 & 21 & $P<0.01$ \\
tet $(B)$ & 12 & 30 & 0.023 \\
$\operatorname{tet}(C)$ & - & 5 & 0.054 \\
$\operatorname{tet}(G)$ & 1 & 5 & 0.205 \\
$\operatorname{tet}(A)+\operatorname{tet}(B)$ & - & 1 & 0.399 \\
$\operatorname{tet}(A)+\operatorname{tet}(C)$ & - & 1 & 0.399 \\
$\operatorname{tet}(B)+\operatorname{tet}(G)$ & 1 & 5 & 0.205 \\
\hline $\operatorname{Num}$
\end{tabular}

Numbers indicated in italics indicate significance difference $(P<0.05)$
aadA2 (two isolates), dfrA12 (two isolates), aadA1-dfrA1 (seven isolates), aadA1-dfrA12 (six isolates), aadA5-dfrA17 (one isolate), aadA1-aadA2-dfrA12 (three isolates) and aadA1-aadA5-dfrA5 (one isolate), were described. The $2.5 \mathrm{~kb}$ amplicon consists of aadA1-aadA2-dfrA12 and aadA1-aadA5-dfrA5 (Table 4).

\section{PFGE analysis of isolates containing class 1 integrons}

The genetic relatedness among the multidrug resistant E. coli isolates carrying integrons was established based on their XbaI-digested chromosomal DNA fragments, and the most commonly detected genotypes are depicted in Table 4 and Fig. 3. Several profiles were observed, with 12-25 discernible DNA fragments from 38 isolates when analyzed by the Dice coefficient method. When an $80 \%$ cut-off band pattern similarity was used, 28 different PFGE clusters were observed, whereas 26 clusters were detected when a $70 \%$ cut-off band pattern similarity was applied. Strong relationships ( $>90 \%$ similarity) were encountered in six clusters constituting 12 isolates sharing the same antibiotics resistance spectrum and resistance gene pattern. For instance, isolates EC174 and EC175 had more than $97 \%$ band pattern similarity, as well as the same antibiotic resistance pattern (TE-S-NA), integron gene cassette arrays (aadA1-dfrA12), and resistance genes [tet(A), sul1 and floR].

\section{Discussion}

Our study investigated the association of class 1 integron carriage, phylogenetic group affiliation and different tetracycline antibiotics resistance patterns in commensal E. coli strains isolated from cattle farms in Korea. All 92 $E$. coli isolates were significantly resistant to tetracycline and oxytetracycline. These findings indicate a widespread application of these antibiotics either for therapeutic purposes or as a supplement for promoting growth, and this continuous exposure to tetracyclines 
Table 4 Characterization of E. coli isolates harboring class 1 integrons and description of their associated gene cassettes

\begin{tabular}{|c|c|c|c|c|c|c|}
\hline $\begin{array}{l}\text { Isolates } \\
\text { No. }\end{array}$ & $3^{\prime} \mathrm{CS}$ & $\begin{array}{l}\text { Cassette amplicons } \\
\text { (bp) }\end{array}$ & $\begin{array}{l}\text { Other resistance gene } \\
\text { pattern }\end{array}$ & $\begin{array}{l}\text { Integron gene cassette } \\
\text { arrays }\end{array}$ & $\begin{array}{l}\text { PFGE } \\
\text { pattern }\end{array}$ & Resistance pattern \\
\hline EC151 & $\begin{array}{l}\text { qacE } \triangle 1- \\
\text { sul1 }\end{array}$ & 1500 & tetA, sul1, sul2, cat1 & aadA1-dfrA1 & B & TE-S-C-SXT \\
\hline EC139 & $\begin{array}{l}\text { qacE } \triangle 1- \\
\text { sul1 }\end{array}$ & 1500 & tetB, tetG, sul1, sul2, cat1, floR & aadA1-dfrA1 & A & TE-S-C-AMP-SXT-CIP-NA \\
\hline EC143 & - & 1500 & tetA, sul3, cm/A, cat1 & $\operatorname{aadA1-dfrA12}$ & U & TE-S-AMP-SXT-CIP-NA \\
\hline EC147 & $\begin{array}{l}\text { qacE } \triangle 1- \\
\text { sul1 }\end{array}$ & 1000 & tetA, sul1 & $\operatorname{aad} A 2$ & $\mathrm{H}$ & TE-S-NA \\
\hline EC152 & $\begin{array}{l}\text { qacE } 17- \\
\text { sul1 }\end{array}$ & 1000 & tet $A$, sul1 & $\operatorname{aadA1}$ & $\mathrm{H}$ & TE-S-AMP-SXT-NA \\
\hline EC153 & $\begin{array}{l}\text { qacE } \triangle 1- \\
\text { sul1 }\end{array}$ & 2500 & tetA, sull & $\operatorname{aadA1-aadA2-dfrA12}$ & $\mathrm{H}$ & TE-S-NA \\
\hline EC155 & $\begin{array}{l}\text { qacE } \triangle 1- \\
\text { sul1 }\end{array}$ & - & tetB, sul1, sul2, cat1 & - & S & TE-S-C-SXT-CIP-NA \\
\hline EC156 & $\begin{array}{l}\text { qacE } \triangle 1- \\
\text { sul1 }\end{array}$ & 1000 & tet $A$, sul1, floR & aadA1 & $\mathrm{H}$ & TE-S-AMP-NA \\
\hline EC157 & $\begin{array}{l}\text { qacE } 17- \\
\text { sul1 }\end{array}$ & 1500 & tetB, sul1, sul2, cat1 & aadA5-dfrA17 & G & TE-S-AMP-SXT-CIP-NA \\
\hline EC159 & $\begin{array}{l}\text { qacE } \triangle 1- \\
\text { sul1 }\end{array}$ & 1500 & tetB, sul1, sul2, cat1 & dfrA12 & $\mathrm{R}$ & TE-S-C-AMP-SXT-CIP-NA \\
\hline EC160 & $\begin{array}{l}\text { qacE } \triangle 1- \\
\text { sul1 }\end{array}$ & 1500 & tetB, sul1, sul2, cat1 & dfrA12 & $\mathrm{R}$ & TE-S-C-AMP-SXT-CIP-NA \\
\hline EC161 & - & - & tet $B, s u l 3, \mathrm{~cm} / A$ & - & Q & TE-S-C \\
\hline EC162 & $\begin{array}{l}\text { qacE } \triangle 1- \\
\text { sul1 }\end{array}$ & 1000 & tetA, sul1, floR & aadAl & N & TE-S-NA \\
\hline EC163 & $\begin{array}{l}\text { qacE } \triangle 1- \\
\text { sul1 }\end{array}$ & 1000 & tetA, sul1, floR & aadA1-dfrAl & L & TE-S-NA \\
\hline EC164 & $\begin{array}{l}\text { qacED1- } \\
\text { sul1 }\end{array}$ & 1000 & tet $A$, sul1, floR & aadA1 & M & TE-S-SXT-NA \\
\hline EC165 & $\begin{array}{l}\text { qacE } \triangle 1- \\
\text { sul1 }\end{array}$ & 1000 & tetB, sul1, sul2, cat1, floR & aadA1 & $\mathrm{T}$ & TE-S-C-AMP-SXT-CIP-NA \\
\hline EC166 & $\begin{array}{l}\text { qacE } \triangle 1- \\
\text { sul1 }\end{array}$ & - & tetB, sul1, sul2, cat1 & - & G & TE-S-C-AMPCIP-NA \\
\hline EC167 & $\begin{array}{l}\text { qacE } \triangle 1- \\
\text { sul1 }\end{array}$ & 2500 & tet $A$, sul1, floR & aadA1-aadA2-dfrA12 & K & TE-S-NA \\
\hline EC172 & $\begin{array}{l}\text { qacE } \triangle 1- \\
\text { sul1 }\end{array}$ & 1000 & tet $A$, sul1, floR & $\operatorname{aadA1}$ & J & TE-S-NA \\
\hline EC173 & $\begin{array}{l}\text { qacE } \triangle 1- \\
\text { sul1 }\end{array}$ & 1000 & tet $A$, sul1, floR & aadA1 & J & TE-S-NA \\
\hline EC174 & $\begin{array}{l}\text { qacE } \triangle 1- \\
\text { sul1 }\end{array}$ & 1000 & tetA, sul1, floR & $\operatorname{aadA1-dfrA12}$ & 1 & TE-S-NA \\
\hline EC175 & $\begin{array}{l}\text { qacE } \triangle 1- \\
\text { sul1 }\end{array}$ & 1000 & tet $A$, sul1, floR & aadA1-dfrA12 & I & TE-S-NA \\
\hline EC176 & qacE $\triangle 1$ & 1000 & tet $B$, sul2, sul3, cmlA, floR & $\operatorname{aadA1}$ & W & TE-S-C \\
\hline EC177 & $\begin{array}{l}\text { qacEDT- } \\
\text { sul1 }\end{array}$ & 1000 & tet $A$, sul1, floR & $\operatorname{aadA1}$ & Y & TE-S-AMP \\
\hline EC178 & $\begin{array}{l}\text { qacE } \triangle 1- \\
\text { sul1 }\end{array}$ & 2500 & tet $A$, sul1, floR & aadA1-aadA5-dfrA5 & Y & TE-S-AMP-NA \\
\hline EC179 & qacE $\triangle 1$ & 2500 & tetB, sul2, sul3, cml1, floR & aad1-aadA2-dfrA12 & $\mathrm{O}$ & TE-S-C \\
\hline EC180 & qacE $\triangle 1$ & 1000 & tetB, sul2, sul3, cml1, floR & $\operatorname{aad} A 2$ & P & TE-S-C \\
\hline EC181 & $\begin{array}{l}\text { qacE } \triangle 1- \\
\text { sul1 }\end{array}$ & 1500 & tetB, sul1, sul2, cat1 & aadA1-dfrA1 & $\mathrm{D}$ & TE-S-C-AMP-SXT-CIP-AMC-NA \\
\hline EC185 & qacE $\triangle 1$-sul1 & 1500 & tet $A$, sull, sul2 & aadA1-dfrA1 & Z & TE-S-AMP-SXT-AMC-NA \\
\hline
\end{tabular}


Table 4 Characterization of E. coli isolates harboring class 1 integrons and description of their associated gene cassettes (Continued)

\begin{tabular}{|c|c|c|c|c|c|c|}
\hline $\begin{array}{l}\text { Isolates } \\
\text { No. }\end{array}$ & $3^{\prime} \mathrm{CS}$ & $\begin{array}{l}\text { Cassette amplicons } \\
\text { (bp) }\end{array}$ & $\begin{array}{l}\text { Other resistance gene } \\
\text { pattern }\end{array}$ & $\begin{array}{l}\text { Integron gene cassette } \\
\text { arrays }\end{array}$ & $\begin{array}{l}\text { PFGE } \\
\text { pattern }\end{array}$ & Resistance pattern \\
\hline EC191 & qacE $\Delta 1$ & 1000 & tetA, sul3, cml1, cat1, floR & aadA1-dfrA12 & A2 & TE-S-C-SXT \\
\hline EC194 & gacE $\triangle 1$-sul1 & 1500 & tetA, sul1, cat1 & aadA1-dfra1 & $\mathrm{F}$ & TE-S-AMP-SXT \\
\hline EC198 & gacE $\triangle 1$-sul1 & 1500 & tet $A$, sul1, cat1 & aadA1-dfra1 & $\mathrm{F}$ & TE-S-AMP-SXT \\
\hline EC209 & qacE $\Delta 1$ & 1000 & tetA, sul3, cml1, cat1, floR & aadA1-dfrA12 & $x$ & TE-S-C-SXT \\
\hline EC230 & qacE $\Delta 1$ & - & tet $A$, sul2, cat1 & - & E & TE-S-C-AMP-SXT-NA \\
\hline EC231 & qacE $\Delta 1$ & - & tet $A$, sul2, cat1 & - & E & TE-S-AMP \\
\hline EC254 & gacE $\Delta 1$-sult & 1000 & tet $A$, sul1, cat1 & aadA1-dfrA12 & V & TE-S-AMP \\
\hline EC258 & sul1 & - & tetA, sul1 & - & $\mathrm{A} 1$ & TE-AMP-SXT \\
\hline EC262 & qacE $\triangle 1$ & 1000 & tet $A$, sul2, cat1, floR & $\operatorname{aadA1}$ & C & TE-S-C-AMP \\
\hline
\end{tabular}

has led to a higher percentage of tetracycline-resistant $E$. coli isolates. The findings of our work were comparable with other observations where a high prevalence of resistance to antimicrobials commonly used with livestock, such as tetracycline and streptomycin were observed in commensal $E$. coli isolated from food-producing animals in South Korea. For instance, Lim et al. [31] observed tetracycline (30.5\%) as the most frequently observed resistance in $E$. coli isolates of cattle origin, and Kang et al. [32] showed that E. coli isolates recovered from swine with diarrhea were highly resistant to streptomycin (99.0\%) and tetracycline (97.1\%); furthermore, the work of Shin et al. [33] also demonstrated that the most prevalent resistance phenotype observed was streptomycin (63.1\%), followed by tetracycline (54.5\%). Tetracycline antibiotics have long been the

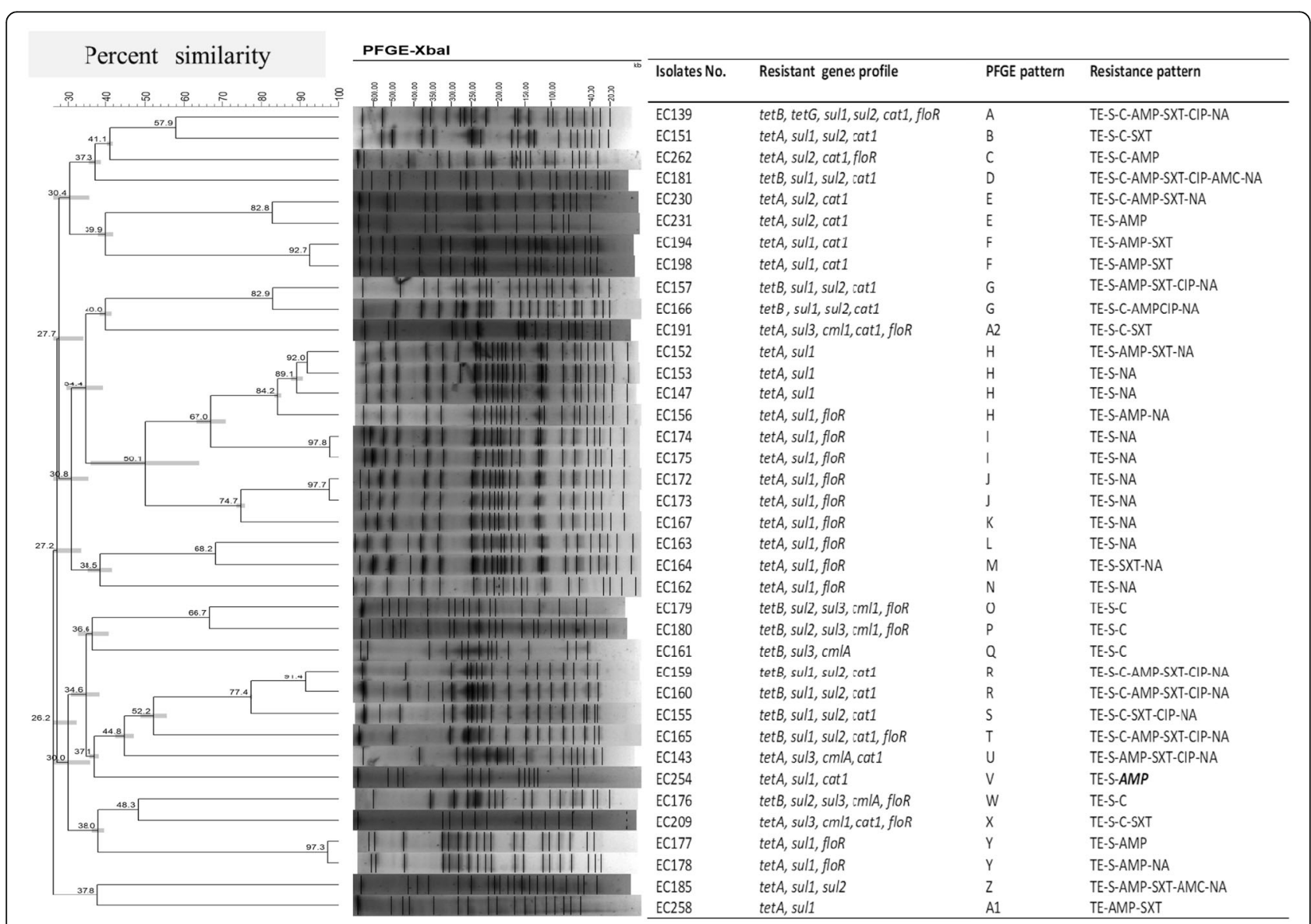

Fig. 3 Genetic relatedness of E. coli isolates with class 1 integrons indicated by Xbal-digested chromosomal DNA 
single most commonly used class of antimicrobial in livestock, accounting for around 50\% of the total amount of antimicrobial consumption both in the USA [34] and Korea [35], and it is, therefore, not surprising to observe tetracycline resistance as the most frequently antimicrobial resistance class in E. coli isolates. As indicated by the animal and plant quarantine agency of Korea (APQA) [36], although gradually decreasing since 2003, tetracyclines still comprise the predominant antibiotics sold for veterinary use.

The investigated isolates were recovered from clinically healthy animals; accordingly, B1 (58.7\%), which is commonly associated with nonpathogenic commensal strains, was the common phylogroup classified. Accordingly, only a single isolate was classified into phylogenetic group B2, which is normally linked with pathogenicity [37]. Moreover, no isolates categorized as B2 carried the intI1 gene, which is similar to the results of a previous study that demonstrated that the B2 phylogroup has a lower tendency to harbor integrons than other phylogroups [16]. In the present study, significant differences in the numbers of isolates with and without integrons were observed $(P<0.01)$ between phylogroups $\mathrm{D}$ and B1. These agree with those of a previous study that demonstrated that strains associated with phylogroups A and B1 tend to carry integrons more often than those associated with $\mathrm{B} 2$ and $\mathrm{D}[10,38]$. In contrast, affiliation with a specific phylogenetic group was not linked to the presence of integrons in $E$. coli strains recovered from river water [39]. This variability is likely because of ecological differences among the sites from which the $E$. coli isolates were recovered that may influence their ability to harbor integron genes.

In the present study, the tet $(A)$ gene was the predominant resistance determinant, followed by the tet(B) gene. There is general agreement regarding the widespread importance of the link between the tet $(A)$ and $\operatorname{tet}(B)$ genes and resistance to tetracycline antibiotics in Enterobacteriaceae as reported by multiple investigators [12, 40, 41]. In this study, isolates having more than one tet gene were also observed in $8.7 \%$ of the strains, which is a common phenomenon in E. coli isolates of cattle origin. Previous studies have shown similar results, in which 3.5\% [42], $5.4 \%$ [43] and $22.2 \%$ [44] of isolates had two tet genes, with only a slight difference in the total number of isolates used between the studies. The acquisition of more than one tet gene by a given strain is attributed to powerful selection pressures due to the high level of tetracycline in the environment rather than to a special selective advantage conferred by the tet genes [12].

There are varying accounts of which tet gene is most frequently reported in different countries. For example, Karami et al. [13] reported that $\operatorname{tet}(B)$ was the most frequently observed (51\%) among commensal $E$. coli strains from Sweden, while Shin et al. [41] and Dessie et al. [45] reported that $\operatorname{tet}(A)$ accounted for $46.5 \%$ and $63.2 \%$ of all tet genes detected in Korea, respectively. A significantly higher frequency of the tet $(A)$ gene $(P<0.01)$ was also observed in isolates with integrons, demonstrating an association between tet $(A)$ carriage and presence of class 1 integrons. This observation has previously been reported by others, who found that intII and tet( $A$ ) coexisted on the same large transferable plasmid or other genetic elements in $E$. coli, validating an established association between tetracycline resistance genes and class 1 integrons [46, 47]. sul1 was identified in $39.1 \%$ of isolates; since it is commonly linked to integrons and transposons as a component of the $3^{\prime}-\mathrm{CS}$, previous studies have similarly reported it among bacteria of the family Enterobacteriaceae [48].

In the present study, $41.3 \%$ of E. coli isolates harbored intI1 gene-encoding class 1 integrons. A comparable result regarding the prevalence of class 1 integrons was previously reported in Korea and other countries; for instance, $40 \%$ of the E. coli isolates carried class 1 integrons in Lithuania [49], $49.8 \%$ in Italy [50], and $27 \%$ in the United States [51], as well as $44 \%$ of the commensal E. coli isolates from poultry in Korea [52]. Non-classical integrons lacking the normal $3{ }^{\prime}-\mathrm{CS}$ were detected in only two class 1 integron-positive isolates. Similar observations were made for intI1-positive $E$. coli isolates that originated from food, animals, and healthy humans [53]. Moreover, $32(84.2 \%)$ of the 38 intI1-positive isolates had variable regions containing gene cassettes. Overall, our analysis showed that the aad and $d f r$ families comprise the majority of class 1 integron gene cassettes, similar to the results reported for $E$. coli originating from beef cattle [38]. In the present study, aadA1-dfrA1 was the most commonly detected combination, which is in agreement with previous reports on isolates recovered from clinical and healthy animals, humans and food samples [27, 49, 52, 54]. Furthermore, $27(71.1 \%)$ of the cassette arrays contained the aadA1 gene, either alone or in combination with other gene cassette arrays that encode aminoglycoside adenyltransferases, which confer resistance to streptomycin/spectinomycin [39]. When we made a comparison between these environmental isolates and clinical isolates from the same region, commensal E. coli isolates from animals mostly carried a single gene cassette, whereas clinical $E$. coli isolates from humans had multigene cassettes [52]. In addition, we found between one and three gene cassettes in a single isolate, which is a distinguishing feature of class 1 integrons in which no more than 6 gene cassettes are carried in the variable region [4].

Based on the results of the XbaI-PFGE, the E. coli isolates carrying class 1 integrons could be categorized into 28 and 26 different PFGE cluster groups when $80 \%$ and 
70\% cut-off band pattern similarities were applied, respectively. In this study, E. coli isolates carrying integrons showed a high degree of polymorphism. This diverse clonal relationship resulted from the horizontal transfer of resistance genes between different strains, rather than a dissemination of a single clonal strain, as previously described [55].

\section{Conclusions}

Due to their carriage of resistant genes and class 1 integrons, commensal E. coli isolates have a significant implication in public health through their ability to disseminate antibiotic resistant genes via contamination of the food chain. A positive association was observed between isolates harboring the $\operatorname{intI1}$ and $\operatorname{tet}(A)$ genes, confirming that isolates containing the $\operatorname{tet}(A)$ gene are more likely to carry class 1 integrons. Likewise, affiliation with phylogroup D was positively associated with the presence of class 1 integrons. Further detailed investigation of the class 1 integron genetic content should be conducted to provide a more complete understanding of the molecular mechanisms responsible for multidrug resistance in E. coli strains. Moreover, the interrelation of integron related resistance genes with other factors should be studied by integrating environmental and veterinary factors and factors associated with the food chain. Accordingly, the resulting advances could have a profound effect on clinical practice, infection control measures and treatment options, both in veterinary and human medicine.

\section{Abbreviations \\ APQA: Animal and plant quarantine agency of Korea; CS: Conserved segment; KFDA: Korean Food and Drug Administration; MIC: Minimum inhibitory concentration; PCR: Polymerase chain reaction; PFGE: Pulsed-field gel electrophoresis; USFDA: United States Food and Drug Administration}

\section{Acknowledgments}

We are grateful to Marie Salomé GABRIAC from IUT Nancy Brabois-Université de Lorraine for her technical support and assistance during laboratory work.

\section{Consent to participate}

Not applicable

\section{Funding}

This study was supported by the "Cooperative research program for agriculture science and technology development (project No. PJ00897001)", Rural Development Administration and Research Institute of Veterinary Science and BK21 PLUS program, Seoul National University, Republic of Korea.

\section{Availability of data and materials}

The datasets generated and/or analyzed during the current study are not publicly available as the work is part of an ongoing PhD research project, but they are available from the corresponding author upon reasonable request.

\section{Authors' contributions}

Conceived the study and participated in its design: KMB SWS HSY. Performed isolation and identification of isolates: KMB. Performed the experiments: KMB. Analyzed the data: KMB HSY. Wrote the paper: KMB. All authors critically reviewed the draft and approved the final manuscript.

\section{Authors' information}

Kuastros Mekonnen Belaynehe: DVM, MSC, PhD research student. Seung Won Shin: DVM, PhD.

Han Sang Yoo: DVM, PhD (Professor of Veterinary Microbiology and director of the Institute of Green Bio Science and Technology, Seoul National University).

\section{Ethics approval}

All animal experimentation was performed according to the guidelines for the care and use of animals approved by the National Institute of Animal Science (permission number: 2013-046).

\section{Consent for publication}

Not applicable.

\section{Competing interests}

The authors declare that they have no competing interests.

\section{Publisher's Note}

Springer Nature remains neutral with regard to jurisdictional claims in published maps and institutional affiliations.

Received: 8 December 2017 Accepted: 22 October 2018

Published online: 12 November 2018

\section{References}

1. Mazel D. Integrons: agents of bacterial evolution. Nat Rev Microbiol. 2006; 4(8):608-20.

2. Cambray G, Guerout AM, Mazel D. Integrons. Annu Rev Genet. 2010;44:141-66.

3. Ponce-Rivas E, Muñoz-Márquez ME, Khan AA. Identification and molecular characterization of class 1 integrons in multiresistant Escherichia coli isolates from poultry litter. Appl Environ Microbiol. 2012;78(15):5444-7.

4. Escudero JA, Loot C, Nivina A, Mazel D. The integron: adaptation on demand. In: Mobile III DNA, editor. MicrobiolSpectrum. USA: American Society of Microbiology; 2015. p. 139-61.

5. Gillings MR. Integrons: past, present, and future. Microbiol Mol Biol Rev. 2014;78(2):257-77.

6. Deng Y, Bao X, Ji L, Chen L, Liu J, Miao J, Chen D, Bian H, Li Y, Yu G. Resistance integrons: class 1, 2 and 3 integrons. Ann Clin Microbiol Antimicrob. 2015;14(1):45.

7. Recchia GD, Hall RM. Gene cassettes: a new class of mobile element. Microbiology. 1995;141(12):3015-27.

8. Carattoli A. Importance of integrons in the diffusion of resistance. Vet Res. 2001:32(3-4):243-59.

9. Stokes HW, Nesbø CL, Holley M, Bahl MI, Gillings MR, Boucher Y. Class 1 integrons potentially predating the association with Tn402-like transposition genes are present in a sediment microbial community. J Bacteriol. 2006; 188(16):5722-30

10. Singh T, Das S, Ramachandran V, Wani S, Shah D, Maroof KA, Sharma A. Distribution of Integrons and phylogenetic groups among Enteropathogenic Escherichia coli isolates from children $<5$ years of age in Delhi, India. Front Microbiol. 2017:8:561.

11. Mazel D, Dychinco B, Webb VA, Davies J. Antibiotic resistance in the ECOR collection: integrons and identification of a novel aad gene. Antimicrob Agents Chemother. 2000;44(6):1568-74.

12. Koo HJ, Woo GJ. Distribution and transferability of tetracycline resistance determinants in Escherichia coli isolated from meat and meat products. Int J Food Microbiol. 2011;145(2):407-13.

13. Karami N, Nowrouzian F, Adlerberth I, Wold AE. Tetracycline resistance in Escherichia coli and persistence in the infantile colonic microbiota. Antimicrob Agents Chemother. 2006;50(1):156-61.

14. Johnson JR, Kuskowski MA, Owens K, Gajewski A, Winokur PL. Phylogenetic origin and virulence genotype in relation to resistance to fluoroquinolones and/or extended-spectrum cephalosporins and cephamycins among Escherichia coli isolates from animals and humans. J Infect Dis. 2003;188(5): 759-68.

15. Bukh AS, Schønheyder HC, Emmersen JM, Søgaard M, Bastholm S, Roslev P. Escherichia coli phylogenetic groups are associated with site of infection and level of antibiotic resistance in community-acquired bacteraemia: a 10 year population-based study in Denmark. J Antimicrob Chemother. 2009; 64(1):163-8. 
16. Skurnik D, Le Menac'h A, Zurakowski D, Mazel D, Courvalin P, Denamur E, Andremont A, Ruimy R. Integron-associated antibiotic resistance and phylogenetic grouping of Escherichia coli isolates from healthy subjects free of recent antibiotic exposure. Antimicrob Agents Chemother. 2005; 49(7):3062-5.

17. Petersen A, Guardabassi L, Dalsgaard A, Olsen JE. Class I integrons containing a dhfrl trimethoprim resistance gene cassette in aquatic Acinetobacter spp. FEMS Microbiol Lett. 2000;182(1):73-6.

18. Belaynehe KM, Shin SW, Park HT, Yoo HS. Occurrence of aminoglycoside modifying enzymes (AMEs) among isolates of Escherichia coli exhibiting high levels of aminoglycoside resistance isolated from Korean cattle farms. FEMS Microbiol Lett. 2017;364:1-9.

19. Clinical and Laboratory Standards Institute (CLSI). Performance standards for antimicrobial susceptibility testing; twenty-third informational supplement. Wayne: Clinical and Laboratory Standards Institute; 2013. M100-S23.

20. Clermont $\mathrm{O}$, Bonacorsi S, Bingen E. Rapid and simple determination of the Escherichia coli phylogenetic group. Appl Environ Microbiol. 2000;66(10): 4555-8.

21. Lanz R, Kuhnert P, Boerlin P. Antimicrobial resistance and resistance gene determinants in clinical Escherichia coli from different animal species in Switzerland. Vet Microbiol. 2003;91(1):73-84.

22. Levy SB, McMurry LM, Barbosa TM, Burdett V, Courvalin P, Hillen W, Roberts MC, Rood JI, Taylor DE. Nomenclature for new tetracycline resistance determinants. Antimicrob Agents Chemother. 1999;43(6):1523-4.

23. Zhao J, Aoki T. Nucleotide sequence analysis of the class $\mathrm{G}$ tetracycline resistance determinant from Vibrio anguillarum. Microbiol Immunol. 1992; 36(10):1051-60

24. Kerrn M, Klemmensen T, Frimodt-Møller N, Espersen F. Susceptibility of Danish Escherichia coli strains isolated from urinary tract infections and bacteraemia, and distribution of sul genes conferring sulphonamide resistance. J Antimicrob Chemother. 2002;50(4):513-6.

25. Kozak GK, Pearl DL, Parkman J, Reid-Smith RJ, Deckert A, Boerlin P. Distribution of sulfonamide resistance genes in Escherichia coli and Salmonella isolates from swine and chickens at abattoirs in Ontario and Quebec, Canada. Appl Environ Microbiol. 2009;75(18):5999-6001.

26. Orman BE, Pineiro SA, Arduino S, Galas M, Melano R, Caffer MI, Sordelli DO, Centrón D. Evolution of multiresistance in nontyphoid Salmonella serovars from 1984 to 1998 in Argentina. Antimicrob Agents Chemother. 2002:46(12): 3963-70.

27. Sáenz Y, Briñas L, Domínguez E, Ruiz J, Zarazaga M, Vila J, Torres C. Mechanisms of resistance in multiple-antibiotic-resistant Escherichia coli strains of human, animal, and food origins. Antimicrob Agents Chemother 2004:48(10):3996-4001.

28. Levesque $C$, Piche L, Larose C, Roy PH. PCR mapping of integrons reveals several novel combinations of resistance genes. Antimicrob Agents Chemother. 1995;39(1):185-91.

29. Wan MT, Chou CC. Class 1 integrons and the antiseptic resistance gene (qacE $\Delta$ ) in municipal and swine slaughterhouse wastewater treatment plants and wastewater-associated methicillin-resistant Staphylococcus aureus. Int J Environ Res Publ Health. 2015;12(16):6249-60.

30. Vali L, Hamouda A, Hoyle DV, Pearce MC, Whitaker LH, Jenkins C, Knight HI, Smith AW, Amyes SG. Antibiotic resistance and molecular epidemiology of Escherichia coli O26, 0103 and 0145 shed by two cohorts of Scottish beef cattle. J Antimicrob Chemother. 2007:59(3):403-10.

31. Lim SK, Lee HS, Nam HM, Cho YS, Kim JM, Song SW, Park YH, Jung SC. Antimicrobial resistance observed in Escherichia coli strains isolated from fecal samples of cattle and pigs in Korea during 2003-2004. Int J Food Microbiol. 2007;116(2):283-6.

32. Kang SG, Lee DY, Shin SJ, Ahn JM, Yoo HS. Changes in patterns of antimicrobial susceptibility and class 1 integron carriage among Escherichia coil isolates. J Vet Sci. 2005;6(3):201.

33. Shin SW, Byun JW, Jung M, Shin MK, Yoo HS. Antimicrobial resistance, virulence genes and PFGE-profiling of Escherichia coli isolates from south Korean cattle farms. J Microbiol. 2014;52(9):785-93.

34. United States Food and Drug Administration (USFDA). Summary Report on Antimicrobials Sold or Distributed for Use in Food-producing Animals US Food and Drug Administration; 2015. p. 2016.

35. Korean Food and Drug Administration (KFDA). Establishment of control system of antibiotics for livestocks. Seoul: Korean Food and Drug Administration; 2004. p. 19-43.
36. Animal and Plant Quarantine Agency of Korea (APQA). Antimicrobial use in livestock and monitoring report of antimicrobial resistance in animal. South Korea: Ministry of Agriculture, Food and Rural Affairs; 2012. p. 1-98.

37. Carlos C, Pires MM, Stoppe NC, Hachich EM, Sato MI, Gomes TA, Amaral LA Ottoboni LM. Escherichia coli phylogenetic group determination and its application in the identification of the major animal source of fecal contamination. BMC Microbiol. 2010;10(1):161.

38. Wu R, Alexander T, Li J, Munns K, Sharma R, McAllister T. Prevalence and diversity of class 1 integrons and resistance genes in antimicrobial-resistant Escherichia coli originating from beef cattle administered subtherapeutic antimicrobials. J Appl Microbiol. 2011;111(2):511-23.

39. Koczura R, Mokracka J, Barczak A, Krysiak N, Kaznowski A. Association between the presence of class 1 integrons, virulence genes, and phylogenetic groups of Escherichia coli isolates from river water. Microb Ecol. 2013;65(1):84-90.

40. Thaker M, Spanogiannopoulos P, Wright GD. The tetracycline resistome. Cell Mol Life Sci. 2010;67(3):419-31.

41. Shin SW, Shin MK, Jung M, Belaynehe KM, Yoo HS. Prevalence of antimicrobial resistance and transfer of tetracycline resistance genes in Escherichia coli isolated from beef cattle. Appl Environ Microbiol. 2015;81: 5560-6.

42. Marshall B, Tachibana C, Levy S. Frequency of tetracycline resistance determinant classes among lactose-fermenting coliforms. Antimicrob Agents Chemother. 1983;24(6):835-40.

43. Sengeløv G, Halling-Sørensen B, Aarestrup FM. Susceptibility of Escherichia coli and Enterococcus faecium isolated from pigs and broiler chickens to tetracycline degradation products and distribution of tetracycline resistance determinants in E. coli from food animals. Vet Microbiol. 2003;95(1):91-101.

44. Bryan A, Shapir N, Sadowsky MJ. Frequency and distribution of tetracycline resistance genes in genetically diverse, nonselected, and nonclinical Escherichia coli strains isolated from diverse human and animal sources. Appl Environ Microbiol. 2004;70(4):2503-7.

45. Dessie HK, Bae DH, Lee YJ. Characterization of integrons and their cassettes in Escherichia coli and Salmonella isolates from poultry in Korea. Poult Sci. 2013:92(11):3036-43.

46. Sunde $M$, Norström $M$. The prevalence of, associations between and conjugal transfer of antibiotic resistance genes in Escherichia coli isolated from Norwegian meat and meat products. J Antimicrob Chemother. 2006; 58(4):741-7.

47. Boerlin P, Travis R, Gyles CL, Reid-Smith R, Lim NJH, Nicholson V, McEwen SA, Friendship R, Archambault M. Antimicrobial resistance and virulence genes of Escherichia coli isolates from swine in Ontario. Appl Environ Microbiol. 2005;71(11):6753-61.

48. Vinué L, Sáenz Y, Somalo S, Escudero E, Moreno MÁ, Ruiz-Larrea F, Torres C. Prevalence and diversity of integrons and associated resistance genes in faecal Escherichia coli isolates of healthy humans in Spain. J Antimicrob Chemother. 2008;62(5):934-7.

49. Povilonis J, Šeputienè V, Ružauskas $M$, Šiugždiniene R, Virgailis M, Pavilonis A, Sužiedèlienè E. Transferable class 1 and 2 integrons in Escherichia coli and Salmonella enterica isolates of human and animal origin in Lithuania. Foodborne Pathog Dis. 2010;7(10):1185-92.

50. Cavicchio L, Dotto G, Giacomelli M, Giovanardi D, Grilli G, Franciosini MP, Trocino A, Piccirillo A. Class 1 and class 2 integrons in avian pathogenic Escherichia coli from poultry in Italy. Poult Sci. 2015;94(6):1202-8.

51. Shaheen BW, Oyarzabal OA, Boothe DM. The role of class 1 and 2 integrons in mediating antimicrobial resistance among canine and feline clinical $E$. coli isolates from the US. Vet Microbiol. 2010;144(3):363-70.

52. Kang HY, Jeong YS, Oh JY, Tae SH, Choi CH, Moon DC, Lee WK, Lee YC, Seol SY, Cho DT. Characterization of antimicrobial resistance and class 1 integrons found in Escherichia coli isolates from humans and animals in Korea. J Antimicrob Chemother. 2005;55(5):639-44.

53. Sáenz Y, Vinué L, Ruiz E, Somalo S, Martínez S, Rojo-Bezares B, Zarazaga M, Torres C. Class 1 integrons lacking gacE $\Delta 1$ and sull genes in Escherichia coli isolates of food, animal and human origins. Vet Microbiol. 2010; 144(3):493-7.

54. Cocchi S, Grasselli E, Gutacker M, Benagli C, Convert M, Piffaretti JC. Distribution and characterization of integrons in Escherichia coli strains of animal and human origin. FEMS Immunol Med Microbiol. 2007;50(1):126-32.

55. Sawant AA, Hegde NV, Straley BA, Donaldson SC, Love BC, Knabel SJ, Jayarao BM. Antimicrobial-resistant enteric bacteria from dairy cattle. Appl Environ Microbiol. 2007;73(1):156-63. 